\title{
PRIMARY ABELIAN GROUPS HAVING ALL HIGH SUBGROUPS ISOMORPHIC
}

\author{
DOYLE O. CUTLER
}

\begin{abstract}
Let $G$ be a primary abelian group such that $G / p^{\omega} G$ is $p^{\omega+n}$-projective for some positive integer $n$, and if $n>1$ then the $(\omega+m)$ th Ulm invariant of $G$ is zero for $0<m<n-1$. We prove that $G$ has the property that all of its high subgroups are isomorphic. An example is given to show that in general the condition on the Ulm invariant is necessary and that this property is not preserved by direct sums.
\end{abstract}

The concept of a high subgroup was introduced into the structure theory of abelian groups by Irwin in [6] where he proved that high subgroups of torsion abelian groups are pure. Irwin and Walker [7] introduced the concept of a $\Sigma$-group, an abelian group in which all high subgroups are direct sums of cyclic groups. They proved (for torsion groups) that if one high subgroup is a direct sum of cyclic groups then they all are and they are all isomorphic. They also raised the question "are all high subgroups of a torsion group isomorphic?" Hill [4] gave an example of a torsion group $G$ with two nonisomorphic high subgroups, and Hill and Megibben [5] set up the machinery to show in some cases just how "bad" things can get. For example, a primary abelian group of cardinality $c=2^{k_{0}}$ with countable basic subgroups may have $2^{c}$ nonisomorphic high subgroups. In [9, p. 366] Irwin asked "under what conditions on $G$ are any two high subgroups of $G$ isomorphic?" (See also [8].)

In this paper we will introduce a class of groups for which any member of the class has the property that all of its high subgroups are isomorphic but not necessarily direct sums of cyclic groups. We will give an example to show that the Ulm invariant restriction imposed on the class is in general necessary, and the direct sum of two groups with the above property need not have this property.

All groups will be additively written $p$-primary abelian groups ( $p$ a fixed but arbitrary prime). If $G$ is a group then $p^{n} G=\left\{p^{n} g \mid g \in G\right\}$ and $p^{\omega} G=\cap_{n<\omega} p^{n} G$. A high subgroup $H$ of $G$ is a subgroup of $G$ maximal with respect to the property $H \cap p^{\omega} G=\{0\}$. A group $G$ is $p^{\omega+n}$-projective if there exists a subgroup $P \subseteq$ $G\left[p^{n}\right]=\left\{g \in G \mid p^{n} g=0\right\}$ such that $G / P$ is a direct sum of cyclic groups. (Or equivalently, see [10], $p^{\omega+n} \operatorname{Ext}(G, A)=0$ for all abelian groups $A$.) If $\alpha$ is an ordinal then by $f_{\alpha}(G)$ we will mean the $\alpha$ th Ulm invariant of $G$. In general the notation and terminology will be that of [2].

Received by the editors August 21, 1980.

1980 Mathematics Subject Classification. Primary 20K10.

Key words and phrases. High subgroup. 
The main result is the following.

THEOREM. Let $G$ be an abelian p-group such that $G / p^{\omega} G$ is $p^{\omega+n}$-projective, and if $n>1$ then $f_{\omega+m}(G)=0$ for $0 \leqslant m<n-1$. Then any two high subgroups of $G$ are isomorphic.

The restriction $f_{\omega+m}(G)=0$ just means that $p^{\omega} G$ has no cyclic summands of order $p^{m+1}$. Since this is true for all $0 \leqslant m<n-1, p^{\omega} G\left[p^{n}\right]$ is a direct sum of cyclic groups each of order $p^{n}$.

Proof of Theorem. Let $H$ and $K$ be high subgroups of $G$. Let $\varphi: G \rightarrow G / p^{\omega} G$ be natural. Then $G\left[p^{n}\right]=H\left[p^{n}\right] \oplus p^{\omega} G\left[p^{n}\right]=K\left[p^{n}\right] \oplus p^{\omega} G\left[p^{n}\right]$ since $f_{\omega+m}(G)$ $=0$ for $0 \leqslant m<n-1$ and $H$ and $K$ are high subgroups of $G$. Thus $\varphi(H)\left[p^{n}\right]=$ $\varphi(K)\left[p^{n}\right]$. (This also follows from Lemma 1.1 of [1].) Note that $\varphi(H)$ and $\varphi(K)$ are $p^{\omega+n}$-projective since they are subgroups of $G / p^{\omega} G$, a $p^{\omega+n}$-projective (Theorem 6.8 in [10]). By Theorem 5 in [3], two $p^{\omega+n}$-projective $p$-groups $H$ and $K$ are isomorphic if there is an isomorphism from $H\left[p^{n}\right]$ into $K\left[p^{n}\right]$ that preserves heights in $H$ and $K$. Since $\varphi(H)$ and $\varphi(K)$ are pure subgroups of $G / p^{\omega} G$ and $\varphi(H)\left[p^{n}\right]=\varphi(K)\left[p^{n}\right]$ the identity map from $\varphi(H)\left[p^{n}\right]$ into $\varphi(K)\left[p^{n}\right]$ preserves heights. Thus $\varphi(H)$ and $\varphi(K)$ are isomorphic. But $\varphi$ restricted to $H$ and $\varphi$ restricted to $K$ are isomorphisms. Thus $H$ and $K$ are isomorphic.

EXAMPLE. The following is an example of two groups $A_{i}, i=1,2$, such that $A_{i} / p^{\omega} A_{i}$ is $p^{\omega+i}$-projective, $p^{\omega} A_{i}=Z\left(p^{i}\right)$ but $A=A_{1} \oplus A_{2}$ is an example of a reduced abelian $p$-group such that $p^{\omega} A \cong Z(p) \oplus Z\left(p^{2}\right), A / p^{\omega} A$ is $p^{\omega+2}$-projective, and $A$ has two nonisomorphic high subgroups.

Let $B_{1}=\bigoplus_{i<\omega}\left\langle x_{2 i+1}\right\rangle$ and $B_{2}=\bigoplus_{i<\omega}\left\langle x_{2 i+2}\right\rangle$ with $o\left(x_{n}\right)=p^{n}$ for $n<\omega$. Let $\bar{B}_{1}$ and $\bar{B}_{2}$ be the torsion completion (with respect to the $p$-adic topology) of $B_{1}$ and $B_{2}$, respectively, and let $\varphi: \bar{B}_{2} \rightarrow \bar{B}_{1}$ be the homomorphism defined by $\varphi\left(x_{2 i+2}\right)=$ $x_{2 i+1}$ for $i<\omega$.

Let $G_{2}$ be a pure subgroup of $\bar{B}_{2}$ containing $B_{2}$ such that $\bar{B}_{2} / G_{2} \simeq Z\left(p^{\infty}\right)$. Let $G_{1}=\varphi\left(G_{2}\right)$. Let $M_{i}, i=1,2$, be a $p^{\omega+i}$-projective $p$-group such that $M_{i}\left[p^{i}\right]=S_{i} \oplus$ $\bar{B}_{i}\left[p^{i}\right]$, the heights of the elements in $\bar{B}_{i}\left[p^{i}\right]$ are the same in $M_{i}$ as in $\bar{B}_{i}$, and $M_{i} / \bar{B}_{i}\left[p^{i}\right]$ is a direct sum of cyclic groups. (For a construction see the proof of Theorem 1 in [3].) This construction yields $M_{i}$ with no elements of infinite height. Let $H_{i}$ be a pure dense subgroup of $M_{i}$ such that $H_{i}\left[p^{i}\right]=S_{i} \oplus G_{i}\left[p^{i}\right]$, and let $\psi_{i}$ : $M_{i} \rightarrow M_{i} / H_{i}$ be natural. Identifying $M_{i} / H_{i}$ with $Z\left(p^{\infty}\right)$, let $A_{i}=\left\{(m, x) \in M_{i} \oplus\right.$ $\left.Z\left(p^{\infty}\right) \mid \psi_{i}(m)=p^{i} x\right\}$, a subdirect sum of $M_{i} \oplus Z\left(p^{\infty}\right)$. Then $p^{\omega} A_{i} \simeq Z\left(p^{i}\right), A_{i} / p^{\omega} A_{i}$ $\cong M_{i}$ (take the projection of $A_{i}$ onto $M_{i}$ ) and $H_{i}$ (identified with $\left\{(h, 0) \mid h \in H_{i}\right.$, $\left.0 \in Z\left(p^{\omega}\right)\right\}$ in the natural way) is a high subgroup of $A_{i}$. Also $H=H_{1} \oplus H_{2}$ is a high subgroup of $A=A_{1} \oplus A_{2}$.

Notice that $A_{i}, i=1,2$, satisfies the conditions of the theorem and hence has the property that all of its subgroups are isomorphic. Also the high subgroups of $A_{i}$ are $p^{\omega+i}$-projective but not $p^{\omega+i-1}$-projective.

We will construct another high subgroup of $A$ that is not isomorphic to $H_{1} \oplus H_{2}$. Write $G_{2}\left[p^{2}\right]=B_{2}\left[p^{2}\right] \oplus \bigoplus_{\alpha \in \Gamma}\left\langle z_{\alpha}\right\rangle$ and $\bar{B}_{1}[p]=G_{1}[p] \oplus\langle z\rangle$. Let $\Lambda \subseteq$ $\Gamma$ such that if we set $L=B_{2}\left[p^{2}\right] \oplus \bigoplus_{\alpha \in \Gamma}\left\langle w_{\alpha}\right\rangle$ where $w_{\alpha}=z_{\alpha}+z$ if $\alpha \in \Lambda$ and 
$w_{\alpha}=z_{\alpha}$ if $\alpha \notin \Lambda$, then $G_{1}[p] \oplus L$ supports no pure subgroup of $\bar{B}_{1} \oplus \bar{B}_{2}$ that is isomorphic to any pure subgroup of $\bar{B}_{1} \oplus \bar{B}_{2}$ supported by $G_{1}[p] \oplus G_{2}\left[p^{2}\right]$. (This is possible since the cardinality of the set of subsets of $\Gamma$ is $2^{c}$, the cardinality of Aut $\bar{B}_{1} \oplus \bar{B}_{2}$ is $c$, and every isomorphism between pure dense subgroups of $\bar{B}$ extends to an automorphism of $\bar{B}$. For an analogous construction see the proof of Theorem 66.4 in [2].) Let $x \in Z\left(p^{\infty}\right)$ such that $\psi_{1}(z)=p x$. Then $(z, x) \in A_{1}$. Considered as a subgroup of $A_{1} \oplus A_{2}$, let $L^{\prime}=B_{2}\left[p^{2}\right] \oplus \bigoplus_{\alpha \in \Gamma}\left\langle u_{\alpha}\right\rangle$ where $u_{\alpha}=z_{\alpha}$ $+(z, x)$ if $\alpha \in \Lambda$ and $u_{\alpha}=z_{\alpha}$ if $\alpha \notin \Lambda$. Let $K$ be a high subgroup of $A$ supported by $H_{1}[p] \oplus S_{2} \oplus L^{\prime}$.

To show that $H$ and $K$ are not isomorphic, suppose the contrary. Let $\lambda: H \rightarrow K$ be an isomorphism. Note that $H /\left(G_{1}[p] \oplus G_{2}\left[p^{2}\right]\right)$ and $K /\left(G_{1}[p] \oplus L^{\prime}\right)$ are direct sums of cyclic groups. Since $\lambda$ is an isomorphism, $H / \lambda^{-1}\left(G_{1}[p] \oplus L^{\prime}\right)$ is a direct sum of cyclic groups. Let

$$
\theta: G_{1}[p] \oplus G_{2}[p] \rightarrow H / \lambda^{-1}\left(G_{1}[p] \oplus L^{\prime}\right)
$$

be natural. Since $G_{1}[p] \oplus G_{2}[p]$ is of finite index in $\bar{B}_{1}[p] \oplus \bar{B}_{2}[p], \theta$ extends to a height nondecreasing homomorphism $\bar{\theta}$ from $\bar{B}_{1}[p] \oplus \bar{B}_{2}[p]$ into a direct sum of cyclic groups $C$ (containing $H / \lambda^{-1}\left(G_{1}[p] \oplus L^{\prime}\right)$ as a pure subgroup). By Lemma 71.1 in [2], there exists an integer $k$ such that $\bar{\theta}\left(p^{k} \bar{B}_{1}[p] \oplus p^{k} \bar{B}_{2}[p]\right)$ is contained in a finite summand of $C$. Thus we can conclude that there is an integer $n$ such that

$$
p^{n} G_{1}[p] \oplus p^{n} G_{2}[p] \subseteq \lambda^{-1}\left(G_{1}[p] \oplus L^{\prime}[p]\right) \text {. }
$$

In a similar manner one can show that there is an integer $m \geqslant n$ such that $p^{m} G_{1}[p] \oplus p^{m} G_{2}\left[p^{2}\right] \subseteq \lambda^{-1}\left(G_{1}[p] \oplus L^{\prime}\right)$. If we choose $m$ to be even then $G_{1}[p] \oplus$ $G_{2}\left[p^{2}\right]=R \oplus p^{m} G_{1}[p] \oplus p^{m} G_{2}\left[p^{2}\right]$ where

$$
R=\bigoplus_{0<2 i+1<m}\left\langle p^{2 i} x_{2 i+1}\right\rangle \oplus \underset{0<2 i<m}{\bigoplus}\left\langle p^{2 i-2} x_{2 i}\right\rangle
$$

(here the $x_{i}$ are those in the definition of $B_{1}$ and $B_{2}$ ).

Next let $\rho=\rho_{1} \oplus \rho_{2}: A_{1} \oplus A_{2} \rightarrow M_{1} \oplus M_{2}$ where $\rho_{i}, i=1,2$, is the projection of $A_{i}$ onto $M_{i}$. Since ker $\rho=p^{\omega}\left(A_{1} \oplus A_{2}\right), \rho \mid G_{1}[p] \oplus L^{\prime}$ is a height preserving isomorphism of $G_{1}[p] \oplus L^{\prime}$ onto $G_{1}[p] \oplus L$. We can write $G_{1}[p] \oplus L=R \oplus p^{m} G_{1}[p] \oplus$ $L^{*}$ such that the heights of the elements of $L^{*}$ in $\bar{B}_{1} \oplus \bar{B}_{2}$ are $>m$. (In the decomposition of $L, z$ has infinite height $\bmod B_{1}[p]$ and the $z_{\alpha}$ have infinite height $\bmod B_{2}\left[p^{2}\right]$.) Thus $\rho \lambda \mid p^{m} G_{1}[p] \oplus p^{m} G_{2}\left[p^{2}\right]$ is a height preserving isomorphism of $p^{m} G_{1}[p] \oplus p^{m} G_{2}\left[p^{2}\right]$ into $p^{m} G_{1}[p] \oplus L^{*}$. Since $p^{m} G_{1}[p] \oplus p^{m} G_{2}\left[p^{2}\right]$ and $p^{m} G_{1}[p]$ $\oplus L^{*}$ both miss $p^{m} B_{1}[p] \oplus p^{m} B_{2}\left[p^{2}\right]$ by a summand isomorphic to $Z(p) \oplus Z\left(p^{2}\right)$ and any height preserving isomorphism of $p^{m} G_{1}[p] \oplus p^{m} G_{2}\left[p^{2}\right]$ into $p^{m} B_{1}[p] \oplus$ $p^{m} B_{2}\left[p^{2}\right]$ extends uniquely to a height preserving automorphism of $p^{m} B_{1}[p] \oplus$ $p^{m} B_{2}\left[p^{2}\right], \rho \lambda$ must map $p^{m} G_{1}[p] \oplus p^{m} G_{2}\left[p^{2}\right]$ onto $p^{m} G_{1}[p] \oplus L^{*}$. Thus as a subgroup of $\bar{B}_{1} \oplus \bar{B}_{2}$ there is a height preserving isomorphism from $G_{1}[p] \oplus G_{2}\left[p^{2}\right]$ onto $G_{1}[p] \oplus L$. Call this isomorphism $\beta$. For $0<n<\omega$, choose $y_{n} \in \bar{B}_{1} \oplus \bar{B}_{2}$ such that $p^{n-i} y_{n}=\beta\left(p^{n-i} x_{n}\right)$ where $i=1$ if $n$ is odd and $i=2$ if $n$ is even. Define

$$
\gamma: \bigoplus_{0<n<\omega}\left\langle x_{n}\right\rangle \rightarrow \underset{0<n<\omega}{\bigoplus}\left\langle y_{n}\right\rangle: x_{n} \rightarrow y_{n} .
$$


Then $\gamma$ extends to an automorphism of $\bar{B}_{1} \oplus \bar{B}_{2}$. Note that $\left(G_{1}[p] \oplus G_{2}\left[p^{2}\right]\right) \cap\left(B_{1}\right.$ $\left.\oplus B_{2}\right)$ is dense in $G_{1}[p] \oplus G_{2}\left[p^{2}\right]$ with respect to the relative $p$-adic topology. Thus the extension of $\gamma$ agrees with $\beta$ on $G_{1}[p] \oplus G_{2}\left[p^{2}\right]$. (This is but a slight generalization of Lemma 5 in [11], the proof being the same.) This contradicts the choice of $L$. Thus $H$ and $K$ are nonisomorphic high subgroups of $A$.

The referee has suggested the following question: Suppose in the Theorem, $\omega$ is replaced by any limit ordinal $\lambda$, and high by $\lambda$ high. Is this new statement true? I wish to thank the referee for his/her helpful comments.

\section{REFERENCES}

1. D. O. Cutler, On the structure of primary abelian groups of countable Ulm type, Trans. Amer. Math. Soc. 152 (1970), 503-518.

2. L. Fuchs, Infinite abelian groups, Vols. 1, 2, Academic Press, New York, 1970 and 1973.

3. $\ldots$, On the $p^{\omega+n}$-projective abelian p-groups, Publ. Math. Debrecen 23 (1976), 309-313.

4. Paul Hill, Certain pure subgroups of primary groups, Topics in Abelian Groups (J. M. Irwin and E. A. Walker, Eds), Scott, Foresman \& Co., Chicago, 1963, pp. 311-314.

5. Paul Hill and Charles Megibben, On primary groups with countable basic subgroup, Trans. Amer. Math. Soc. 124 (1966), 49-59.

6. J. M. Irwin, High subgroups of abelian torsion groups, Pacific J. Math. 11 (1961), 1375-1384.

7. J. M. Irwin and Elbert A. Walker, On N-high subgroups of abelian groups, Pacific J. Math 11 (1961), 1363-1374.

8. J. M. Irwin, Carol Peercy and Elbert Walker, Splitting properties of high subgroups, Bull. Soc. Math. France 90 (1963), 185-192.

9. J. M. Irwin and E. A. Walker (Eds.), Problems on abelian groups, Topics in Abelian Groups, Scott, Foresman \& Co., Chicago, 1963, pp. 365-368.

10. R. J. Nunke, Purity and subfunctors of the identity, Topics in Abelian Groups (J. M. Irwin and E. A. Walker, Eds.), Scott, Foresman \& Co., Chicago, 1963, pp. 121-171.

11. Fred Richman, Extensions of p-bounded groups, Arch. Math. 21 (1970), 449-454.

Department of Mathematics, University of California, Davis, California 95616 